\title{
Patrones biogeográficos de los tenebriónidos epigeos (Coleoptera: Tenebrionidae) del Área Natural Protegida Península Valdés, Argentina: implicaciones para su conservación
}

\section{Biogeographic patterns of epigean tenebrionids (Coleoptera: Tenebrionidae) from Protected Natural Area Península Valdés, Argentina: implications for its conservation}

\author{
Rodolfo Carrara $^{1}$, Germán H. Cheli ${ }^{2}$ y Gustavo E. Flores ${ }^{1}$ \\ ${ }^{1}$ Laboratorio de Entomología, Instituto Argentino de Investigaciones de las Zonas Áridas (IADIZA, CRICYT), Casilla de Correo 507, 5500 Mendoza, \\ Argentina. \\ ${ }^{2}$ Unidad de Investigación Diversidad, Sistemática y Evolución, CENPAT-CONICET, Bvd. Brown 2915, 9120 Puerto Madryn, Chubut, Argentina \\ 凶rcarrara@mendoza-conicet.gob.ar
}

\begin{abstract}
Resumen. Se realizó un estudio sobre los patrones biogeográficos de los tenebriónidos epigeos (Coleoptera: Tenebrionidae) que habitan el Área Natural Protegida Península Valdés (Chubut, Argentina), basado en datos de colecciones y material recolectado en viajes a la zona. Se presenta una lista exhaustiva de las especies en la que se consigna el grado de adaptación a la aridez de cada una de ellas; un análisis de la calidad del inventario y la estimación de la riqueza total de especies; una evaluación de los patrones de distribución y riqueza, y la identificación de áreas de significativa mayor riqueza o hotspots. Los resultados indicaron que 25 especies con diferente grado de adaptación a la aridez habitan la península, aunque se estima que la riqueza total del área debería ser de 27 . Además, se reconoció que los rangos de distribución de los tenebriónidos siguen una distribución log-normal y que sólo 3 especies son endémicas. Espacialmente, la riqueza se distribuyó de forma agregada en la península y se determinaron 10 hotspots. Finalmente, considerando que en la península Valdés están permitidas actividades productivas que originan una importante degradación del hábitat, se examina la implicancia de los resultados para la conservación de los tenebriónidos epigeos.
\end{abstract}

Palabras clave: lista de especies, adaptaciones a la aridez, endemismos, riqueza de especies, efecto península, efecto del dominio medio, degradación de hábitat, áreas prioritarias de conservación.

\begin{abstract}
We conducted a study of the biogeographic patterns of epigean tenebrionids (Coleoptera: Tenebrionidae) that inhabit the Protected Natural Area Península Valdés (Chubut, Argentina), based on data obtained from collections and collecting trips. We present a comprehensive species list, where we detail the degree of adaptation to aridity shown by each species, an analysis of the quality of the inventory, an estimation of total species richness, and an evaluation of distribution and richness patterns, with the identification of areas of significantly greater richness or "hotspots". The results indicated that 25 species with different degrees of adaptation to aridity inhabit the peninsula, although it was estimated that the total richness of the area should be 27 species. In addition, it was recognized that the species range sizes follow a log-normal distribution and that only three species are endemic. The spatial distribution of richness was aggregate within the peninsula and we can identify 10 "hotspots". Finally, considering that in the Península Valdés are allowed productive activities that cause significant habitat degradation, we discuss the implicances that have these findings for epigean tenebrionids conservation.
\end{abstract}

Key words: species list, adaptations to aridity, endemism, species richness, peninsula effect, mid domain effect, habitat degradation, priority areas for conservation.

\section{Introducción}

El Área Natural Protegida Península Valdés (ANP-PV) constituye una de las mayores unidades de conservación de ecosistemas áridos de América del Sur y fue declarada patrimonio de la humanidad por la UNESCO en 1999. A pesar de la importancia que tiene este sitio para la con-

Recibido: 16 septiembre 2010; aceptado: 13 diciembre 2010 servación ambiental, el conocimiento de su biodiversidad es parcial. De acuerdo con las investigaciones que se han desarrollado en el ANP-PV, existe un importante inventario de la flora (Bertiller et al., 1981; Beeskow et al., 1987) y fauna de vertebrados (Pardiñas et al., 2003; Roig et al., 2009; Nabte et al., 2009), pero es escaso el conocimiento de la fauna de invertebrados que la habita. Particularmente es necesario aumentar el estudio sobre la diversidad de invertebrados, ya que estos organismos dominan en casi 
todos los ambientes sobre la Tierra y proveen diversos beneficios y servicios al ecosistema (Cheli et al., 2010).

La forma de mejorar el conocimiento de los invertebrados que habitan el ANP-PV, además de desarrollar estudios que releven las especies presentes en ella, es realizar trabajos que profundicen sobre sus patrones biogeográficos, ya que esta información contribuye al desarrollo de acciones precisas para la protección de la diversidad (Peterson et al., 2000). En los ambientes áridos, uno de los grupos más representativos de invertebrados es el de los insectos (Polis, 1991); sin embargo, para el ANP-PV existe un único estudio que ha tenido como objeto su diversidad (Cheli et al., 2010). En esa investigación se describió la composición y estructura de la comunidad de insectos que habitan la península a escala local, por lo que nos ofrece un conocimiento preliminar del número total de especies que habitan el ANP-PV pero no provee información acerca de los patrones regionales de distribución y riqueza de los mismos.

De acuerdo con los resultados obtenidos por Cheli et al. (2010), se pudo observar que una de las familias de insectos con mayor abundancia en número de especies e individuos dentro del ANP-PV fue Tenebrionidae (Coleoptera). Este hallazgo es semejante a los resultados de Flores et al. (2004) y Cepeda-Pizarro et al. (2005a, b) en los que esta familia de coleópteros también ha sido una de las más abundantes en otros ecosistemas áridos de América del Sur, desempeñando un papel clave en el ciclado de nutrientes y como indicadores de procesos de degradación de hábitat (Cepeda-Pizarro et al., 2005a). Específicamente, existe una clara distinción entre aquellos tenebriónidos de hábitos arborícolas y los de hábitos terrestres (Aalbu et al., 2002a), siendo los terrestres (i.e., epigeos) los que conforman el grupo numérica y funcionalmente dominante de invertebrados de los ambientes áridos (Koch, 1961; Crawford y Seely, 1987; Cepeda-Pizarro et al., 2005b). Esta dominancia de los tenebriónidos epigeos se debe a que han desarrollado adaptaciones fisiológicas, etológicas y morfológicas para sobrevivir a condiciones de aridez (Cloudsley-Thompson, 2001). Las principales de estas adaptaciones son los hábitos nocturnos o, cuando son diurnos, el ocultarse entre el sustrato durante las horas de mayor radiación solar; los tamaños corporales grandes para aumentar la longevidad y el control de su homeostasis; la unión íntima entre los élitros a lo largo de la sutura y también con los esternitos abdominales y la presencia de una cavidad subelitral que ayuda al control de la pérdida de agua por los espiráculos. Esta cavidad subelitral se desarrolló como consecuencia de la pérdida del segundo par de alas, que los convierte en ápteros (Zachariassen, 1996; Cloudsley-Thompson, 2001). El apterismo les confiere, por un lado, una alta exposición a predadores, por lo que representan uno de los principales recursos alimenticios de muchos vertebrados de zonas desérticas (Flores, 1998; Donadio et al., 2004; Superina et al., 2009) y por otro lado, un bajo poder de dispersión, por lo que son uno de los grupos de insectos con mayor endemicidad de los ambientes áridos (Watt, 1974; Flores, 1998); por esta característica fueron utilizados para delimitar áreas de endemismo en el sur de América del Sur (Roig-Juñent y Flores, 2001; Morrone et al., 2002; Domínguez et al., 2006; Morrone, 2006). Debido a estas evidencias, el conocimiento de las especies de tenebriónidos epigeos que habita una región y sus patrones de distribución son de suma importancia para ser considerados en políticas de conservación.

Con base en datos provenientes de 40 años de recolecciones en la península Valdés, alojados en varias colecciones biológicas de museos y de varios viajes de recolección efectuados a la zona desde 2005, los objetivos de este trabajo son: 1), presentar la primera lista exhaustiva de tenebriónidos epigeos que habitan el ANP-PV, consignando el grado de adaptación a la aridez de cada especie y analizando sus patrones de distribución, con la identificación de las especies endémicas y la distribución regional de las que viven en la península y no son endémicas; 2), estimar la calidad del inventario y la riqueza total de especies y evaluar el patrón espacial de la riqueza observada y 3), valorar la existencia de áreas con mayor riqueza significativa respecto de la riqueza observada (i.e., hotspots de diversidad), y con base en los resultados obtenidos considerar las implicancias que éstos tienen en acciones de conservación. Es importante notar que si bien la lista de especies de tenebriónidos epigeos que se provee en este trabajo puede incrementarse en el futuro, esta clase de investigaciones representan un buen punto de partida para entender la evolución de los ensambles de insectos y para establecer comparaciones con patrones peninsulares de otros taxones (Johnson y Ward, 2002).

\section{Materiales y métodos}

Área de estudio. El ANP-PV está ubicada al nordeste de la provincia de Chubut en la República Argentina entre los $42^{\circ} 05^{\prime} \mathrm{S}$ y $42^{\circ} 53^{\prime} \mathrm{S}$ y los $63^{\circ} 05^{\prime} \mathrm{O}$ y $64^{\circ} 37^{\prime} \mathrm{O}$. El área consiste en una meseta de $3625 \mathrm{~km}^{2}$ rodeada por las aguas del océano Atlántico; geológicamente, está formada por sedimentos marinos oligomiocénicos y una cubierta de sedimentos eólicos con gravas cuartáricas (Súnico et al., 1994; Haller et al., 2001). La configuración actual del relieve se habría originado durante el Pleistoceno debido a la conjunción de la densidad de fallas existentes en el basamento paleozoico, la movilización de sedimentos por erosión hidráulica y eólica y la acción de intensos vientos de la región que habrían provocado la deflación de los 
golfos Nuevo y San José (Kostadinoff, 1992; Haller et al., 2001). El clima es árido y tiene una temperatura media anual de $14^{\circ} \mathrm{C}$, con temperaturas máximas y mínimas absolutas de $44^{\circ} \mathrm{C}$ y $-12^{\circ} \mathrm{C}$ y precipitaciones medias anuales de $175 \mathrm{~mm}$ en la zona costera, oscilando en el interior entre 200 y 225 mm (Súnico et al., 1994).

El paisaje general es una planicie con áreas deprimidas (salinas) y cordones medanosos en su tercio austral (Rostagno, 1981). La fisonomía dominante es una estepa arbustiva de Chuquiraga avellanedae Lorentz (Asteraceae), Ch. erinacea ssp. hystrix D. Don (Asteraceae), Condalia microphylla Cav. (Rhamnaceae), Brachyclados megalanthus Speg. (Asteraceae), Lycium chilense Miers ex Bertero (Solanaceae), Schinus polygamus (Cav.) Cabrera (Anacardiaceae) y Prosopidastrum globosum (Gillies ex Hook. et Arn.) Burkart (Mimosaceae). El tapiz de gramíneas presenta una cobertura variable, siendo las especies más comunes Nassella tenuis (Phil.) Barkworth (Poaceae), Piptochaetium napostaense (Speg.) Hack. (Poaceae) y Poa ligularis Nees ex Steud. (Poaceae) (Bertiller et al., 1981). Entre las hierbas se encuentran Hoffmannseggia trifoliata Cav. (Fabaceae), Paronychia chilensis DC. (Caryophyllaceae), Daucus pusillus Michx. (Apiaceae) y Plantago patagonica Jacq. (Plantaginaceae). Hacia el sur, la estepa arbustiva da paso a una estepa herbácea de Sporobolus rigens (Trin.) E. Desv. (Poaceae), N. tenuis, Pappostipa speciosa (Trin. et Rupr.) Romaschenko var. speciosa (Poaceae), Panicum urvilleanum Kunth (Poaceae), Poa lanuginosa Poir. (Poaceae) y P. napostaense acompañados de Ch. avellanedae y Hyalis argentea D. Don ex Hook et Arn (Asteraceae) (Bertiller et al., 1981; León et al., 1998). Base de datos. Las colecciones en las cuales está basada la lista de tenebriónidos epigeos del ANP-PV son: Instituto Argentino de Investigaciones de las Zonas Áridas, Mendoza; Museo Argentino de Ciencias Naturales Bernardino Rivadavia, Buenos Aires; Museo de La Plata, La Plata, Buenos Aires; Instituto Superior de Entomología Dr. Abraham Willink, Fundación e Instituto Miguel Lillo, San Miguel de Tucumán; Centro Nacional Patagónico (CENPAT), Puerto Madryn, Chubut, todas de Argentina. Como ha sido anotado en la introducción, la mayoría de los tenebriónidos terrestres son ápteros; sin embargo, existen pocas especies que poseen el segundo par de alas y no tienen los élitros fusionados pero desarrollan su vida como insectos terrestres que viven debajo de troncos y piedras (Aalbu et al., 2002a). Teniendo en cuenta estas características, en este estudio se tomó la decisión de incluir tenebriónidos epigeos tanto ápteros como con alas.

Se revisaron 2757 ejemplares, los cuales fueron identificados de acuerdo con sus datos de colecta y localización geográfica, considerando la latitud y longitud del sitio (georreferenciación). Cabe destacar que los ejemplares que se encontraron con la misma información fueron considerados como duplicados y se ingresaron a la base de datos como un solo registro. Si bien esta decisión hizo disminuir a menos del $15 \%$ la cantidad de registros de especies en el área (en total quedaron 378 registros válidos), se aceptó como lo apropiado para evitar seudorreplicas en los datos.

Para identificar las especies se siguieron las revisiones y claves de Kulzer $(1955,1958,1963)$ y Flores (1999, 2004), y se compararon con material depositado en la colección entomológica de IADIZA. La clasificación propuesta por Bouchard et al. (2005) se tomó como base para asignar las especies a tribus y subfamilias.

Luego, utilizando el sistema de información geográfica (GIS) mediante el programa DIVA-GIS (Hijmans et al., 2005), se procedió a dividir el ANP-PV en celdas geográficas de $0.05^{\circ} \times 0.05^{\circ}$ (i.e., aproximadamente $25 \mathrm{~km}^{2}$ ), en las que se volcaron los registros georreferidos de las especies. La resolución de considerar el tamaño de celda de $25 \mathrm{~km}^{2}$ se debió a que el ANP-PV está dividido en promedio por parcelas o cuadros de ese tamaño (Organismo Provincial de Turismo, 2001) y que, de acuerdo con lo que se conoce, pueden ser interpretadas como una unidad de manejo para la conservación. Toda celda en la cual no se contabilizó registro de especies alguno fue eliminada del análisis, por lo que quedaron un total de 60 celdas válidas.

A partir de los registros asignados a cada celda se construyeron 2 matrices: una de incidencias y otra de frecuencias. Ambas matrices están representadas por las especies en las filas $(i)$ y por las celdas geográficas en las columnas $(j)$, pero difieren en los elementos $(i, j)$ que contienen: la matriz de incidencias considera como elemento los datos de presencia o ausencia de especies en cada celda (i.e., valores 1 y 0 respectivamente); en cambio, la matriz de frecuencias, considera como elementos $(i, j)$ el número de ejemplares registrados por especie por celda. Estas matrices fueron utilizadas para extraer tanto la información de riqueza de especies por celda geográfica $\left(\alpha_{i}\right)$, mediante la suma de los elementos de cada columna de la matriz de incidencia, y el esfuerzo de recolección por celda (h), mediante la suma de los elementos de la columna de la matriz de frecuencias (como en Garcillán et al., 2003). Adaptaciones a la aridez. Los tenebriónidos epigeos que habitan el ANP-PV se agruparon según su capacidad para soportar las condiciones desérticas considerando los 3 grupos propuestos por Sánchez-Piñero y Aalbu (2002): los tenebriónidos muy altamente adaptados a los ambientes áridos, que están caracterizados por la presencia de élitros fusionados, cavidad subelitral, ausencia de glándulas defensivas y condición áptera; los altamente adaptados, caracterizados en la misma forma que los anteriores pero con la presencia de glándulas defensivas, y los menos adaptados, que están caracterizados por la presencia del 
segundo par de alas y provistos a menudo de glándulas defensivas.

Intervalos geográficos. Se estimó el intervalo de distribución de cada especie dentro de la península como el número de celdas ocupado por una especie, para esto se sumaron los elementos por fila de la matriz de incidencia. Con base en los intervalos de distribución del conjunto total de especies del ANP-PV, se calculó el área de distribución promedio de las especies, se identificaron las que presentaron una distribución geográfica restringida (considerando como tales aquellas cuyos intervalos de distribución fueron menores al primer cuartil) y las que poseen amplia distribución geográfica (considerando como tales, aquellas cuyos intervalos de distribución fueron mayores al tercer cuartil). Además, se analizó la distribución estadística de los intervalos de distribución de las especies de tenebriónidos, comparándolos con una distribución log-normal, bajo la hipótesis nula de que los intervalos geográficos de distribución, como estimadores de las abundancias poblacionales, están distribuidos de forma log-normal (Preston 1962; Garcillán et al., 2003). Para este último análisis se utilizó el módulo nortest (Groos, 2006) dentro del programa estadístico $R$ (R Development Core Team [2010]). Finalmente, con base en datos complementarios extraídos de las colecciones, fue identificada la distribución regional de las especies que habitan en el ANP-PV para conocer la existencia de especies endémicas, o de especies con distribución en alguna de las 2 provincias biogeográficas más cercanas a la península, Patagonia o Monte, o de ambas, o de especies cuya distribución geográfica se extiende más allá de la Patagonia y Monte.

Evaluación de la calidad del inventario y estimación de la riqueza de especies. Debido a que el relevamiento de los tenebriónidos epigeos es incompleto, resulta necesario hacer una evaluación de la calidad del inventario existente para estimar cuán completo y fiable es. En este caso, la evaluación se hizo mediante la utilización de curvas de acumulación de especies que relacionan la aparición de nuevas especies con el inventario, de acuerdo con una medida de esfuerzo de muestreo (Jiménez-Valverde y Hortal, 2003). Una vez obtenida la curva de acumulación, se evaluó la calidad del inventario, ajustando la ecuación de Clench a dicha curva (Soberón y Llorente, 1993; JiménezValverde y Hortal, 2003). Esta ecuación se define como:

$$
S_{n}=a n /(1+b n) \quad \text { eq. } 1,
$$

donde $S_{n}$ es el número medio de especies en una área, $n$ es la unidad de esfuerzo de muestreo, siendo $a$ un parámetro que representa la tasa de incremento de nuevas especies al comienzo del inventario y $b$ un parámetro relacionado con la forma de la curva (véase Jiménez-Valverde y Hortal,
2003). A partir de la eq. 1 se puede, por un lado, derivar una función para estimar la proporción de especies registrada con el esfuerzo realizado, lo que permite a su vez evaluar la calidad del inventario $\left[S_{\text {prop }}=S_{\text {obs }} /(a / b)\right]$, donde $S_{\text {prop }}$ es la proporción de la riqueza inventariada, $S_{\text {obs }}$ es la riqueza observada; y, por otro, derivar una función para estimar la riqueza total de especies esperadas en el área $\left[S_{e}=a / b\right]$, donde $S_{e}$ es la riqueza esperada.

En este estudio se considera como unidad de esfuerzo cada una de las unidades de muestreo $(n=60$ celdas) en las cuales se distribuyen los 378 registros de tenebriónidos epigeos. Debido a que distintas formas de curvas de acumulación pueden obtenerse de acuerdo con el orden de entrada de las unidades de esfuerzo, resulta necesario suavizar la curva. Para construir la curva de acumulación de especies de tenebriónidos se utilizó el programa EstimateS 7.52 (Colwell, 2005) que permitió replicar 100 veces las curvas de acumulación y obtener una curva resultante del promedio de esas 100 (i.e., curva suavizada). Para el ajuste y estimación de los parámetros de la ecuación de Clench se utilizó el programa estadístico $R$ con la función $n l s$ dentro del paquete stats (R Development Core Team, 2010). Sumado a la ecuación de Clench, se estimó la riqueza aplicando $\mathrm{ChaO}_{2}$ que utiliza algoritmos estadísticos derivados del método de captura-recaptura (Chao, 1987) mediante el programa EstimateS.

Patrones de riqueza de especies. Primero se evaluó si el patrón observado de riqueza de especies $(\alpha)$ en el ANP-PV se distribuye en forma agrupada, uniforme o azarosa en el espacio. Para esto se aplicó la prueba de cociente entre varianza y media (CVM; Cox y Lewis, 1966) por el que, bajo el supuesto de que la riqueza se distribuye según una distribución Poisson, se considera que si el valor de CVM es igual a 1, la riqueza se distribuye de forma aleatoria, si el valor de CVM es significativamente $<1$, la riqueza se distribuye regularmente y que si el valor de CVM es $>1$, la riqueza está distribuida de forma agrupada. Para evaluar si el valor resultante de la prueba de CVM es significativamente diferente de 1 , será aplicada una prueba de $t$ bajo la fórmula: $\left[t=\left(O_{(C V M)}-E_{(C V M)}\right) / S E_{(C V M)}\right]$; donde $O_{(C V M)}$ es el valor observado del cociente entre varianza y media, $E_{(C V M)}$ es el valor esperado del cociente entre varianza y media según una distribución de Poisson (i.e., 1) y $S E_{(C V M)}$ es el error estándar del cociente entre varianza y media.

También se evalúa el ajuste a los datos de 2 modelos comúnmente utilizados como explicaciones del patrón de riqueza de especies en penínsulas. Por un lado, se consideró el modelo del "efecto península" (EP) y por otro, el de "efecto del dominio medio" (EDM). El EP postula una disminución de la riqueza de especies desde la base de la península hasta su punta (Simpson, 1964) debido al equilibrio entre procesos de inmigración-extinción de 
individuos de diferentes especies que decrece a mayores distancias a lo largo de la península y al tamaño del área que previene extinciones (MacArthur y Wilson, 1967). Por el contrario, la hipótesis EDM postula la influencia de los límites geográficos de la península como un factor estocástico que determina la riqueza de especies (Colwell y Lees, 2000), por lo que bajo este supuesto se espera encontrar una mayor riqueza de especies en las celdas del centro de la península que en las celdas de la periferia (i.e, las celdas cercanas al mar; Ferrer-Castán y Vetaas, 2005).

Para evaluar estos 2 modelos se utilizó el programa BioGeoSim (Gotelli et al., 2007) que simula el emplazamiento estocástico de las especies a lo largo de un área heterogénea. Este programa, a partir de un dominio grillado estima la riqueza de especies esperada bajo repetidas simulaciones de la ubicación de los rangos de distribución de esas especies (Rahbek et al., 2007). Finalmente, esta riqueza esperada es ajustada mediante una regresión estándar a la riqueza observada. Debido a que las simulaciones se basan en un valor asignado a cada grilla del dominio, es necesario traducir los modelos EP y EDM en valores para cada una de las celdas de $25 \mathrm{~km}^{2}$ en las que fue dividida la península. Para asignar el valor a cada celda según EP, se consideró el efecto inverso que tiene la distancia de cada celda al continente $\left(d_{i j}\right)$ y el efecto directo que tiene el tamaño del área de cada celda $\left(a_{i j}\right)$ sobre la riqueza de especies; por lo que el valor de cada celda $\left(P_{i j}\right)$ será $P_{i j}=\left(1 / d_{i j}\right) a_{i j}$. Para el EDM, al ser la riqueza de especies influenciada únicamente por los límites geográficos las probabilidades de cada grilla son constantes, i.e., $P_{i j}=1$ (Rahbek et al., 2007). En total, los efectos de estas hipótesis fueron evaluados mediante la repetición de 500 simulaciones, considerando el total de dominio, con un algoritmo de dispersión contiguo. Debido a que el efecto de la autocorrelación espacial en los datos de riqueza de especies por celda hace que muchos de ellos no sean independientes, para estimar la significancia en la relación entre riqueza observada y esperada se aplicó la corrección de Dutilleul (1993) mediante el programa SAM (Rangel et al., 2010). La estimación tanto de la distancia entre las celdas a la base de la península y el área disponible en cada celda se hizo mediante el uso de GIS.

Hotspots de diversidad. Se identificaron las celdas dentro del ANP-PV que presentaron la mayor riqueza de especies de tenebriónidos $(\alpha)$. Para conseguir este objetivo se siguió la metodología propuesta por Garcillán et al. (2003), donde los datos de riqueza observada fueron comparados estadísticamente con la riqueza esperada, de acuerdo con la corrección de 2 factores: el esfuerzo dispar en la recolección de especies por celda y la variación en el tamaño de área por celda. Por lo que las celdas con una significativa mayor riqueza que la esperada bajo la corrección de estos 2 factores fueron consideradas hotspots de diversidad de tenebriónidos.

Para corregir la riqueza observada por el efecto del esfuerzo de recolección se ajustó una función lineal que considera el número de especies observado en cada celda por la cantidad de registros encontrados en ella:

$$
s_{i}^{*}=k+d p_{i} \quad \text { eq. } 2,
$$

donde $s_{i}{ }^{*}$ es la riqueza corregida por esfuerzo de recolección, $\rho$ que se define como $1-(s / h)$; donde $s$ es número de especies encontrados en cada celda y $h$ es el número de registros encontrados en cada celda, y $k \mathrm{y} d$ son constantes. En esta regresión lineal se ajusta la riqueza de las 60 celdas $\left(\alpha_{i}\right)$ de la península contra la redundancia del esfuerzo de colección de cada celda $\left(\rho_{i}\right)$.

Para corregir la riqueza observada por el área disponible en cada celda se consideró la función de potencia de la relación especies-área (Preston, 1962; MacArthur y Wilson, 1967):

$$
S=c A^{z} \quad \text { eq. } 3,
$$

donde $S$ es el número de especies presentes en un área de tamaño $A, z$ y $c$ son constantes. A partir de esta expresión deriva una función que considere la riqueza esperada luego de corregir por el esfuerzo de colección, y que estime un tamaño de estándar de área $\left(A_{0}\right)$ y use ese valor como corrector de la riqueza observada por celda:

$$
\alpha_{i^{*}}=s_{i}^{*}\left(\frac{A_{i}}{A_{0}}\right)^{z} \quad \text { eq. } 4,
$$

donde, $\alpha_{i^{*}}$ es la riqueza esperada, $s_{i}^{*}$ es la riqueza corregida por el esfuerzo de recolección de una celda de tamaño estándar conocida $\left(A_{0}\right)$ y $A_{i}$ es el área de cada celda. El valor de área estándar $\left(A_{0}\right)$ se obtiene a partir del promedio

$$
A_{0}=\left(\frac{\sum s_{i}^{*} A_{i}^{z}}{\sum s_{i}^{*}}\right)^{(1 / z)} \quad \text { eq. } 5 \text {, }
$$

ponderado exponencialmente de todas las celdas consideradas en el estudio:

Para obtener el valor de $A_{0}$ es necesario estimar primero el valor de $z$, por lo que para obtenerlo se seleccionó al azar desde 1 hasta 30 celdas sumando el área y la riqueza obtenida para crear 30 muestras; luego, a partir de estas muestras, se ajustó la versión logarítmica de la eq. $3(\log S=\log c+z \log A)$ y se estimó el exponente $z$ con el cual se resolvió la eq. 5. Finalmente, se identificó 
cuáles valores de la riqueza observada fueron significativamente mayores que los $\alpha_{i^{*}}$ esperados, por medio de la eq. 6, constituyéndose en hotspots de diversidad. Para esto, se calcularon los residuos de Pearson $(E)$ entre los valores observados y predichos de riqueza de especies.

$$
\left(\varepsilon=\left(\alpha_{i}-\alpha_{i^{*}}\right) / \sqrt{\alpha_{i^{*}}}\right) .
$$

Debido a que los valores de riqueza de especies son conteos discretos con una distribución esperada de Poisson, estos residuos deberían comportarse asintóticamente como desviaciones normales, por lo que su significancia pudo ser evaluada desde la distribución normal (Garcillán et al., 2003).

\section{Resultados}

En total se encontraron 25 especies de tenebriónidos epigeos que habitan el ANP-PV de las cuales sólo 3 poseen el segundo par de alas y no tienen los élitros fusionados, y las restantes fueron especies ápteras (Cuadro 1). De las 10 subfamilias de Tenebrionidae (Bouchard et al., 2005) sólo 2 están representadas en el ANP-PV: Pimeliinae, que reúne el $80 \%(n=20)$, y Tenebrioninae, el $20 \%(n=5)$ del total de especies de la península.

Las 25 especies encontradas están agrupadas en 8 tribus, 6 de Pimeliinae: Nycteliini que representa el 36\% de las especies $(n=9)$, Praocini el 28\% $(n=7)$; Edrotini, Physogasterini, Stenosini y Trilobocarini, cada una con 4\% $(n=1)$; 2 de Tenebrioninae: Scotobiini con $16 \%$ $(n=4)$, y Opatrini con $4 \%(n=1)$. Respecto a los 15 géneros presentes en la península, 9 están representados sólo por 1 especie y 6 poseen más de 1 especie (Nyctelia Latreille y Praocis Eschscholtz con 4 especies, y Patagonogenius Flores, Calymmophorus Solier, Leptynoderes Solier y Emmallodera Blanchard, cada uno con 2 especies). De acuerdo con el grado de adaptación a la aridez propuesto por Sánchez-Piñero y Aalbu (2002) (Cuadro 1) se reconoció que el $72 \%$ de las especies están muy altamente adaptadas (comprendiendo las especies agrupadas en las tribus Nycteliini, Physogasterini, Praocini y Stenosini), el 16\% están altamente adaptadas (especies agrupadas en la tribu Scotobiini) y el $12 \%$ menos adaptadas (especies de las tribus Edrotini, Trilobocarini y Opatrini).

En promedio, el intervalo de distribución de cada especie fue de 11.24 celdas (equivalentes a $281 \mathrm{~km}^{2}$ ), hubo 8 especies de distribución restringida y 6 de amplia distribución (Cuadro 1). Los intervalos de los tenebriónidos epigeos no demostraron ser significativamente distintos de los de una distribución log-normal (Kolmogorov-Smirnov normal test, $D=0.1, p=0.53$ ).
Examinando la distribución regional de las especies que habitan la península se pudo conocer que sólo 3 especies son endémicas del ANP-PV, 8 especies sólo habitan la provincia biogeográfica del Monte, 7 únicamente la provincia biogeográfica de la Patagonia, 3 habitan tanto Monte como Patagonia (Cabrera y Willink, 1980; Morrone, 2006) y 4 tienen una distribución que se extiende más allá de del Monte y Patagonia (véase Cuadro 1). Debe notarse que de estas 4 especies una es áptera (Nyctelia nodosa Germar) y 3 con alas (Hylithus tentyroides Lacordaire, Salax lacordairei Guérin-Méneville y Blapstinus punctulatus Solier). La especie áptera se extiende hasta Uruguay (Kulzer, 1963) y las aladas hasta Chile, al oeste de la cordillera de Los Andes (Peña, 1966). Se piensa que los géneros de estas especies aladas no fueron afectados por el levantamiento de la cordillera (Flores y Pizarro-Araya, 2006).

La evaluación de la calidad del inventario de tenebriónidos epigeos del ANP-PV demostró que este es completo y altamente fiable. Esta aseveración resultó del ajuste significativo del modelo Clench $\left(S_{n}=3.72 n / 1+0.14 n, R^{2}=0.99 \%, p<0.0001\right)$ que a partir de la estimación de sus parámetros demostró que las especies relevadas conforman el $91.89 \%$ del total de especies conocidas para esta área. Se estimó que la riqueza total de tenebriónidos epigeos para el ANP-PV es de 27. La estimación coincidió con el resultado encontrado por el método $\mathrm{ChaO}_{2}$ (Fig. 2). Esta concordancia en la riqueza total por diferentes métodos otorga consistencia al resultado encontrado.

La riqueza promedio de tenebriónidos por celda observada en el ANP-PV fue de 4.68. El valor encontrado por la prueba de CVM fue de 1.37 siendo significativamente mayor que $1(p<0.001)$. Este resultado sugiere que la distribución espacial de la riqueza está agrupada en la península; sin embargo, ninguno de los 2 modelos propuestos explicó los patrones de riqueza, ya que ambos mostraron una baja y no significativa correlación entre la riqueza observada $\left(S_{o}\right)$ y esperada $\left(S_{e}\right)$; i.e., $\operatorname{EDM}\left(S_{o}=1.50-0.12 S_{e(E D M)}, R=-0.01, p=0.67\right)$ y EP $\left(S_{o}=2.51-0.87 S_{e(E P)}, R=-0.28, p=0.62\right)$. Estos resultados indican que según el ajuste al modelo EDM, la riqueza de especies no está agrupada en las celdas del centro de la península más que en las de la periferia (Fig. 3a). Además indican que según el ajuste al modelo EP no existe un agrupamiento de la riqueza en la base de la península más que en la punta; por el contrario, existe la tendencia no significativa a encontrar un agrupamiento de la riqueza de especies en la punta más que en la base; i.e., por el valor negativo de la pendiente (Fig. 3b). Resumiendo, la riqueza de especies de tenebriónidos en el ANP-PV no estuvo influenciada por el efecto aleatorio de los límites geográ- 
Cuadro 1. Lista de especies con su posición taxonómica, distribución regional y grado de adaptación a la aridez de los tenebriónidos epigeos que habitan el Área Natural Protegida - Península Valdés, Argentina

\begin{tabular}{|c|c|c|c|c|}
\hline Subfamilia & Tribu & Especie & Distribución & Adaptados a la aridez \\
\hline \multirow{20}{*}{ Pimeliinae } & Edrotini & Hylithus tentyroides* $(+)$ & Monte, Chile norte, Chaco & Menos adaptado \\
\hline & \multirow[t]{9}{*}{ Nycteliini } & Epipedonota cristallisata $(+)$ & Monte, Patagonia (Argentina) & Muy altamente adaptado \\
\hline & & Mitragenius araneiformis $(+)$ & Patagonia (Argentina y Chile) & Muy altamente adaptado \\
\hline & & Nyctelia circumundata & Monte & Muy altamente adaptado \\
\hline & & Nyctelia darwini (-) & Patagonia (Argentina y Chile) & Muy altamente adaptado \\
\hline & & Nyctelia dorsata $(+)$ & Monte y Patagonia (Argentina) & Muy altamente adaptado \\
\hline & & Nyctelia nodosa $(+)$ & $\begin{array}{c}\text { Monte, Chaco, Pampa (Argentina y } \\
\text { Uruguay) }\end{array}$ & Muy altamente adaptado \\
\hline & & Patagonogenius collaris & Monte & Muy altamente adaptado \\
\hline & & Patagonogenius quadricollis(-) & Patagonia (Argentina) & Muy altamente adaptado \\
\hline & & Psectracelis sulcicollis & Monte, Patagonia (Argentina) & Muy altamente adaptado \\
\hline & \multirow{8}{*}{$\begin{array}{c}\text { Physogasterini } \\
\text { Praociini }\end{array}$} & Pimelosomus sphaericus(-) & Monte & Muy altamente adaptado \\
\hline & & Calymmophorus patagonicus(-) & Monte & Muy altamente adaptado \\
\hline & & Calymmophorus peninsularis(-) & Endémica & Muy altamente adaptado \\
\hline & & Plathestes kuscheli & $\begin{array}{l}\text { Patagonia } \\
\text { (Argentina) }\end{array}$ & Muy altamente adaptado \\
\hline & & Praocis (Orthogonoderes) argentina(-) & Patagonia (Argentina) & Muy altamente adaptado \\
\hline & & Praocis (Hemipraocis) sellata granulipennis & Monte & Muy altamente adaptado \\
\hline & & Praocis (Hemipraocis) sellata peninsularis & Endémica & Muy altamente adaptado \\
\hline & & Praocis (Hemipraocis) sp.(-) & Endémica & Muy altamente adaptado \\
\hline & Stenosini & Ecnomoderes bruchi & Monte & Muy altamente adaptado \\
\hline & Trilobocarini & Salax lacordaire ${ }^{*}(-)$ & Monte, Chile norte, Chaco & Menos adaptado \\
\hline \multirow[t]{5}{*}{ Tenebrioninae } & Opatrini & Blapstinus punctulatus* & Monte, Chile Central, Chaco & Menos adaptado \\
\hline & \multirow[t]{4}{*}{ Scotobiini } & Emmallodera crenatocostata crenatocostata & Patagonia (Argentina) & Altamente adaptado \\
\hline & & Emmallodera hirtipes $(+)$ & Monte & Altamente adaptado \\
\hline & & Leptynoderes strangulata & Monte & Altamente adaptado \\
\hline & & Leptynoderes tuberculata & Patagonia (Argentina) & Altamente adaptado \\
\hline
\end{tabular}

Especie: * presenta alas, (-) de distribución restringida, $(+)$ de amplia distribución en la península, las especies de Monte son todas endémicas de Argentina.

ficos ni por el balance entre inmigración-extinción, siendo otros factores los que gobiernan la distribución agrupada de los tenebriónidos en el área.

El efecto de la intensidad de recolección por celdas mostró ser significativo, ya que la redundancia en la recolección se comportó de forma significativa como el modelo lineal esperado $\left(S^{*}=4.08+3.80 \rho, R=0.30\right.$, $p=0.019)$, y el cálculo del tamaño de $A_{0}$ fue de 22.58 $\mathrm{km}^{2}$. Luego de corregir la redundancia de recolección y el tamaño de área se encontraron 10 celdas que presentaron significativamente una mayor riqueza que la observada (Fig. 4 a). De acuerdo con su ubicación, fue posible notar que estas celdas estuvieron situadas cerca o incluyeron parte de la costa (i.e., en la periferia de la península). En estas celdas, identificadas como hotspots, estuvo representado el $88 \%$ de las especies que fueron relevadas en el ANP-PV (i.e., 22 de las 25 especies relevadas). 


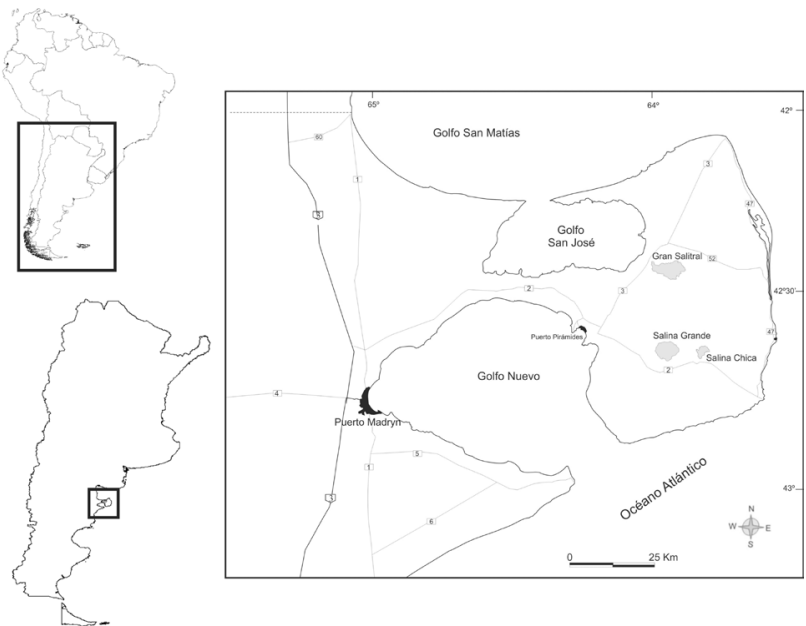

Figura 1. Ubicación geográfica de la península Valdés, Argentina.

a

b
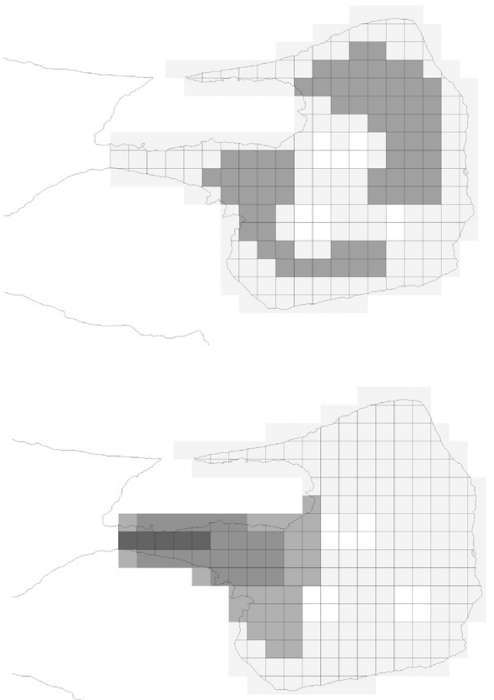

c

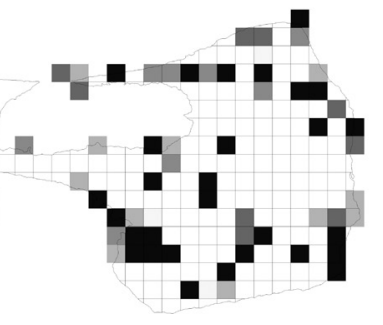

Riqueza de especies

$\square 0$ - 1
$\square 1-2$
$\square 2-3$
$\square 3-4$
$\square-11$

Figura 3. Modelos espacialmente explícitos utilizados para evaluar el patrón espacial de distribución de riqueza de especies de tenebriónidos epigeos en la península Valdés: a, riqueza esperada por unidad de muestreo bajo la hipótesis del efecto del dominio medio; b, riqueza esperada por unidad de muestreo bajo la hipótesis del efecto península, y c, riqueza observada en este estudio por unidad de muestreo.

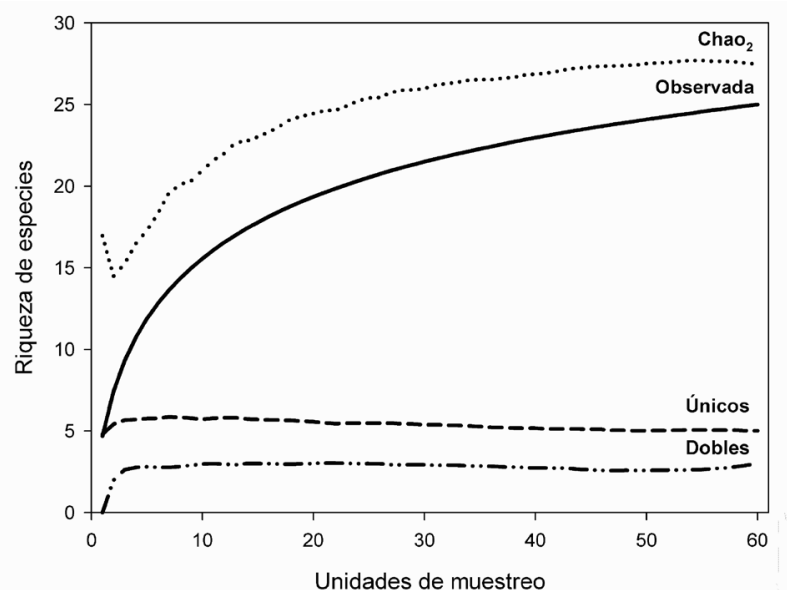

Figura 2. Curvas de acumulación de riqueza de especies por unidad de muestreo; donde Observada, curva de riqueza de especies observada en península Valdés; $\mathrm{ChaO}_{2}$, curva de riqueza estimada a partir del estimador $\mathrm{Chao}_{2}$; Únicos, cantidad de especies que sólo están presentes en una unidad de muestreo y Dobles, cantidad de especies que sólo están presentes en 2 unidades de muestreo.
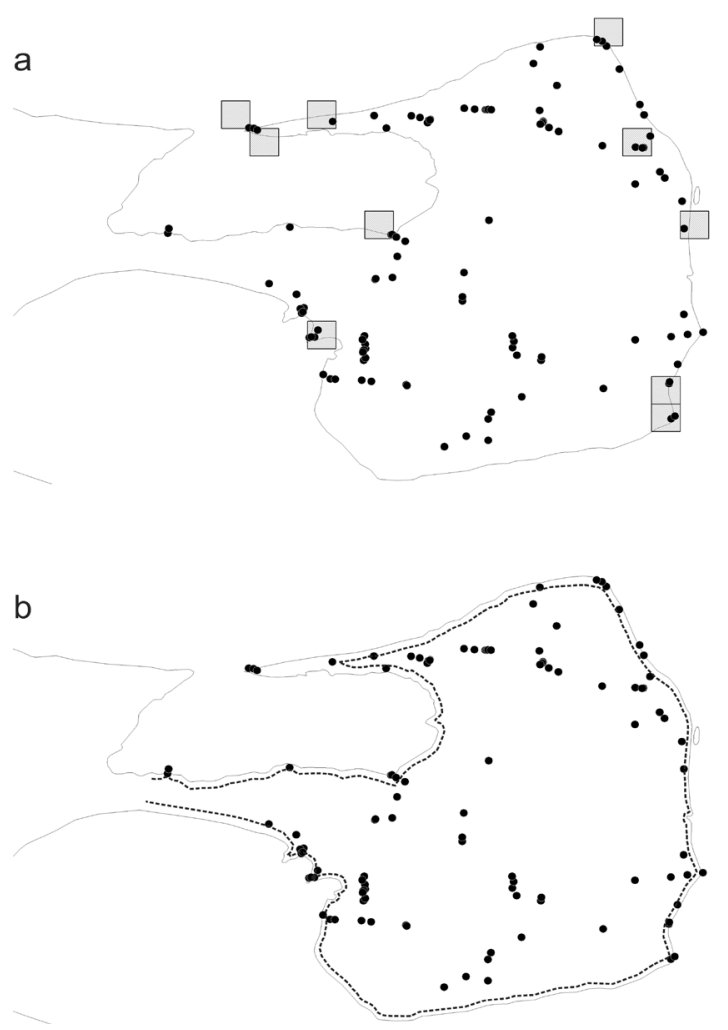

Figura 4. Península Valdés con la distribución espacial de los datos de tenebriónidos epigeos, mostrando: a, celdas consideradas como de mayor riqueza significativa de diversidad de tenebriónidos epigeos (hotspots) y b, línea punteada que representa aproximadamente la zona intangible de $5 \mathrm{~km}$ propuesta por los autores. 


\section{Discusión}

La lista de 25 especies provista en este trabajo releva casi en su totalidad las especies de tenebriónidos epigeos que habitan el ANP-PV. El 80\% de estas especies están agrupadas en 3 tribus: Nycteliini, Praocini y Scotobiini, lo que concuerda con Sackmann y Flores (2009) quienes observaron que estas 3 tribus representaron el $82 \%$ de la comunidad de tenebriónidos epigeos. Previamente, Kuschel (1969) afirmó que los tenebriónidos de la estepa patagónica están representados principalmente por estas 3 tribus neotropicales. La presencia en la península Valdés del $88 \%$ de tenebriónidos epigeos, altamente adaptados a la aridez, es comparable con los resultados obtenidos para tenebriónidos de la península e islas de baja California (Sánchez-Piñero y Aalbu, 2002). Los hallazgos dan cuenta de cómo el estrés hídrico ambiental condiciona la presencia de tenebriónidos, por lo que sólo pueden sobrevivir en estos ambientes las especies que han desarrollado estrategias contra la pérdida de agua. Esta afirmación se sustenta también con la escasa presencia de especies menos adaptadas a la aridez, probablemente debido a la alta susceptibilidad que presentan a la desecación, por conservar sus alas y no poseer los élitros fusionados ni la cavidad subelitral (Sánchez-Piñero y Aalbu, 2002).

La riqueza de tenebriónidos epigeos encontrada en el ANP-PV sugiere que, en relación con su superficie, esta área aloja un importante número de especies del total de tenebriónidos epigeos que habitan la República Argentina. Esto surge de la lista de especies por géneros provista por Flores (1998), a partir de la cual se pudo determinar que en Argentina (con una superficie de 3 $761274 \mathrm{~km}^{2}$ ), habitan 264 especies de tenebriónidos epigeos, por lo que en el ANP-PV (con $3625 \mathrm{~km}^{2}$ ), habita aproximadamente el $10 \%$ de esos tenebriónidos. Gracias a esta investigación, el número de especies conocidas de tenebriónidos epigeos de la península aumentó más de 3 veces ( $n=8$; Cheli et al., 2010); no obstante, de acuerdo con el ajuste del modelo Clench a los datos de la curva de acumulación, si se aplicara mayor esfuerzo de recolección en el área serían encontradas aún más especies. En otra investigación, también a escala regional, efectuada en el límite oeste de la estepa patagónica Argentina (a unos $600 \mathrm{~km}$ al oeste del ANP-PV; Sackmann y Flores, 2009), se encontraron 28 especies de tenebriónidos epigeos. Aunque esta riqueza es casi similar a la observada en península Valdés, es interesante que sólo 3 especies se comparten entre ambas regiones (Blapstinus punctulatus e Hylithus tentyroides, ambas aladas, y Mitragenius araneiformis Curtis especie áptera). Este alto recambio de especies entre un área y otra sugiere la existencia de distintos procesos históricos que sumados a factores ecológicos pueden haber tenido lugar en la evolución espacial de la Patagonia (Morrone et al., 2002).

La distribución espacial de la riqueza de tenebriónidos epigeos por celda dentro de la península está agregada y esta agregación está causada por factores ambientales distintos al equilibrio entre inmigración-extinción. La ausencia del EDM sostiene que el patrón de riqueza observado lo causan factores ambientales (Ferrer-Castán y Vetaas, 2005). Si bien el ajuste del EDM puede estar influenciado por no incluir en el análisis únicamente especies endémicas, como fue propuesto originalmente por Colwell y Hurtt (1994), este resultado es confiable porque el EDM también se aplica a especies no endémicas que habitan una península y que no están influenciadas por las poblaciones de la misma especie que existen fuera de ella (Ferrer-Castán y Vetaas, 2005). La falta de significancia del EP y la tendencia a encontrar más riqueza de tenebriónidos en la punta que en la base de la península, implica que el equilibrio entre los procesos de colonización desde el continente y el efecto del área previniendo extinciones no actúan como factores dinámicos que promueven la riqueza observada. Jenkins y Rinne (2008) sugieren que puede existir un efecto dual o reverso en el EP si existen colonizaciones desde el continente hacia otras zonas de la península (e.g., a la punta de la península). En este caso, se desestima que el patrón de riqueza tenga relación alguna con el equilibrio entre inmigración extinción, debido a que los tenebriónidos epigeos tienen bajo poder de dispersión al ser principalmente ápteros (i.e., únicamente pueden colonizar la península desde el continente y no volando).

De acuerdo con lo observado, se sugiere que una posible causa detrás del patrón de riqueza puede estar relacionada con el efecto de la variación en la calidad de suelo y vegetación de la península. Particularmente, la península Valdés presenta 2 tipos de suelos superficiales dominantes: arenosos y gravosos (Rostagno, 1981; Bouza et al., 2007), cuya dureza condiciona, por un lado, la vegetación que lo habita (Bertiller et al., 1981; Rostagno, 1981), y por lo tanto la disponibilidad de alimento para los tenebriónidos, y por otro lado, su reproducción, ya que su ovipostura es subterránea (Tschinkel y Doyen, 1980; Flores, 1998). Apoyando esta sugerencia, se observó que el $100 \%$ de las especies del ANP-PV habitan en áreas costeras que presentan suelos arenosos y vegetación herbáceo-arbustiva; mientras que sólo un $68 \%$ fueron encontradas en áreas del centro con suelos gravosos y vegetación arbustiva. En diversos trabajos ha sido discutida la influencia del suelo y la vegetación como variables que afectan los ensambles de tenebriónidos (Botes et al., 2007 y referencias allí citadas), por lo que un análisis que considere a ambos como limitantes de la distribución de tenebriónidos epigeos del ANP-PV puede conducir a interesantes resultados. 
Los intervalos de distribución de los tenebriónidos demostraron seguir una distribución log-normal, indicando que dentro de la península la mayor parte de ellos habita áreas reducidas y la menor parte ocupa áreas extensas. A menudo se ha considerado que el intervalo de distribución de una especie es consecuencia directa de su abundancia poblacional (Brown, 1995); así en la península, hay muchas especies poco abundantes y pocas muy abundantes. Esta información es particularmente importante en conservación, ya que a partir del conocimiento de la distribución de frecuencias que siguen los intervalos de distribución de las especies y sus abundancias poblacionales es posible considerar cuáles son raras y por lo tanto tienen mayor posibilidad de extinción (Preston, 1962; Lande, 1993). Incluso, al concentrarse la mayoría de los intervalos de distribución de los tenebriónidos en pocas celdas bien definidas como hotspots, es posible considerar estas áreas como prioritarias en conservación.

El patrón de distribución regional de los tenebriónidos epigeos que habitan la península indica que la alta diversidad encontrada en el ANP-PV puede estar relacionada con las características fitogeográficas que ésta posee. Debido a que en la península Valdés confluyen elementos de vegetación representativos tanto de la provincia biogeográfica de la Patagonia como del Monte (Bertiller et al., 1981), también confluyen tenebriónidos epigeos cuya distribución regional se extiende principalmente en estas 2 provincias (el 84\% de los tenebriónidos). Esto sugiere que la riqueza de tenebriónidos del ANP-PV está influenciada positivamente por la coexistencia de organismos que han experimentado distintas historias evolutivas. Estas evidencias, desde un punto de vista zoogeográfico, podrían contribuir a esclarecer el debate que aún existe sobre la identidad biogeográfica de la península Valdés; ya que para algunos autores, esta área corresponde a la provincia biogeográfica del Monte (Cabrera y Willink, 1980; Roig, 1998; Roig et al., 2009), para otros a la provincia de la Patagonia (Soriano, 1956; Súnico et al., 1994; Morrone, 2006) y para otros más constituye una unidad particular definida como ecotono Monte-Patagonia (León et al., 1998; Elissalde et al., 2002). De acuerdo con los resultados obtenidos, la distribución regional de los tenebriónidos apoyaría la idea de que biogeográficamente la península constituiría un ecotono entre el Monte y la Patagonia, aunque para confirmar esta hipótesis se requieren estudios específicos.

Los 3 taxones endémicos encontrados (Flores et al., 2011) caracterizan a la península como un área de porcentaje de endemicidad relativamente bajo (i.e., $12 \%$ de endemicidad). Este hallazgo puede ser comparado con otras áreas donde se ha reconocido que el aislamiento del continente influye positivamente sobre el número de especies endémicas de un área (i.e., penínsulas e islas; Simpson, 1964; MacArthur y Wilson, 1967; Huston, 1994); en este contexto, el $12 \%$ de endemicidad encontrado en este estudio resultó ser sustancialmente menor al encontrado en la península de Baja California (53\%; Aalbu et al., 2002b) e islas del mar de Cortés (23\%; Sánchez-Piñero y Aalbu, 2002); pero fue mayor al encontrado en el archipiélago de Choros (7\%; Alfaro et al., 2009). Una explicación para este bajo porcentaje de endemismos en el ANP-PV puede estar relacionada con su historia ambiental. El área donde está emplazada la península Valdés data del Mioceno tardío (9-6.8 millones de años antes del presente; Dozo et al., 2010), cuando era un cabo o punta que ingresaba al océano Atlántico sin la presencia de los golfos San José y Nuevo (Fig. 1). Es posible que desde su génesis el área fuera colonizada por los tenebriónidos adaptados a la aridez, ya que por la distribución actual de tribus se puede inferir que habitaban las zonas desérticas de América del Sur austral, previo al levantamiento de Los Andes, de 14-11 millones de años antes del presente (Kulzer, 1955; Flores y PizarroAraya, 2006); y porque las condiciones atmosféricas en la región fueron propicias para la subsistencia de estos organismos ya que durante el Mioceno tardío comenzó a tener un clima seco (Barreda y Palazzesi, 2007) que fue acentuándose hasta lograr las condiciones de aridez que hoy presenta (Hartley, 2003). Posiblemente bajo este escenario de conquista temprana del área por parte de los tenebriónidos epigeos, las poblaciones de especies estaban conectadas entre sí, pero durante el Pleistoceno (aproximadamente 1 millón de años antes del presente) el cabo comenzó a modificarse debido a la formación de 2 depresiones, que a partir del aumento definitivo del nivel del mar en el final de la última gran glaciación (19 mil años antes del presente) conformaron los 2 golfos, originando así la península Valdés (Codignotto, 2008). A partir de ese momento pudo ocurrir un aislamiento entre las poblaciones de las mismas especies dentro y fuera de la península por la formación del istmo, aunque probablemente es muy reciente como para originar procesos de especiación y la generación de un alto grado de endemicidad dentro de península Valdés. Esta suposición, además, se basa en la ausencia de un equilibrio dinámico en la península entre colonizaciones y extinciones (supuestos del EP).

Implicancias para la conservación. Aunque la conservación de la diversidad de tenebriónidos es importante en sí misma, porque forma parte de la biodiversidad global, también lo es porque estos insectos tienen valor como recurso biológico en el funcionamiento de los ecosistemas, e.g., participan en la descomposición de la materia orgánica, son importantes constituyentes de la cadena 
trófica, sobre todo en ambientes áridos (Flores, 1998) y, al ser vulnerables a las modificaciones del hábitat, pueden ser considerados como indicadores de cambios ambientales (Marcuzzi, 1951). Si bien la península presenta un número bajo de especies endémicas, muchas de las especies de tenebriónidos epigeos que la habitan representan endemismos de Argentina (76\%), de Argentina y Chile $(20 \%)$, y de Argentina y Uruguay (4\%), lo que indica que al conservar los tenebriónidos de península Valdés también se conservan especies únicas que sólo habitan estos países. Además, la lista de tenebriónidos epigeos provista en esta investigación constituye el primer eslabón de la cadena de acciones que promueven la conservación, ya que en ella se presenta de forma confiable y precisa la identidad y relaciones taxonómicas de las especies que es la información necesaria para desarrollar programas de monitoreo (Groom et al., 2006).

Debido a que el ANP-PV fue clasificado como categoría VI entre las unidades de conservación de la Unión Mundial para la Naturaleza, dentro de ella está permitido el manejo del recurso turístico y ganadero. Se ha realizado un estudio sobre el efecto del pastoreo en la comunidad de insectos del ANP-PV, aún inédito (Cheli, 2009), y a la fecha no existen publicaciones acerca del impacto que tienen estas actividades sobre la comunidad de tenebriónidos de la península.

Los autores de este estudio consideramos que tanto el pastoreo como el turismo pueden afectar negativamente la diversidad. La presencia de 360000 turistas por año en las costas de la península Valdés (www.estadistica.chubut.gov.ar), donde está la mayor riqueza de tenebriónidos, produce la alteración del hábitat, excesiva cantidad de basura y ocasionales incendios. De la misma forma, el disturbio por pastoreo produce un marcado deterioro del hábitat (Gardner et al., 1995; Molina et al., 1999; Deslippe et al., 2001; González-Megías et al., 2004). A nuestro entender, profundizar los estudios de cómo estas 2 actividades en la península afectan la diversidad de tenebriónidos es prioritaria, ya que la degradación del hábitat conlleva a una reducción de los nichos disponibles para estos coleópteros y, por consiguiente, puede promover la extinción local de las especies (Lawton y May, 1995).

Como la totalidad de tenebriónidos epigeos habitan zonas cercanas al mar, se pueden reforzar las acciones de conservación focalizándose no sólo en los hotspots sino en esas zonas de la península. De hecho, como en el plan de manejo del ANP-PV las costas de la península se han considerado como zona intangible, en pos de proteger los vertebrados marinos y terrestres, recomendamos ampliar esta zona de intangibilidad $5 \mathrm{~km}$ desde la costa hacia adentro de la península, como una acción que protegería a todas las especies de tenebriónidos epigeos (Fig. 4b).

\section{Agradecimientos}

A Lucía A. Puppo Byant, por su aporte en el diseño de las figuras, $\mathrm{Al}$ Ministerio de Comercio Exterior, Turismo e Inversiones (Dirección General de Conservación de Áreas Protegidas) y al Ministerio de Industria, Agricultura y Ganadería (Dirección de Fauna y Flora Silvestre) de la Provincia de Chubut (Argentina), por otorgar los permisos de recolección y alojamiento en el ANP-PV. Al proyecto de la Fundación BBVA (España): «Diseño de una red de reservas para la protección de la biodiversidad en América del Sur Austral, utilizando modelos predictivos de distribución con taxones hiperdiversos», por el uso de la base de datos de Tenebrionidae (Coleoptera). Este estudio fue financiado por el Consejo Nacional de Investigaciones Científicas y Técnicas (CONICET, Argentina) y por el proyecto PIP 112-200801-00162 (CONICET, Argentina). Finalmente, a la Fundación Vida Silvestre Argentina por su colaboración en la logística de la campaña 2010 en el ANP-PV.

\section{Literatura citada}

Aalbu, R. L., C. A. Triplehorn, J. M. Campbell, K. W. Brown, R. A. Somerby y D. B. Thomas. 2002a. Family 106. Tenebrionidae Latreille 1802. In American beetles, vol. 2. Polyphaga: Scarabaeoidea through Curculionoidea, R. H. Arnett, M. C. Thom.as, P. E. Skelley, and J. H. Frank (eds.). CRC, Boca Raton, Florida. p. 463-509.

Aalbu, R. L., G. E. Flores y C. A. Triplehorn. 2002b. Tenebrionidae. In Biodiversidad, taxonomía y biogeografía de artrópodos de México: hacia una síntesis de su conocimiento, J. Llorente-Bousquets y J. J. Morrone (eds.) Facultad de Ciencias, Universidad Nacional Autónoma de México, México, D. F. p. 499-512.

Alfaro, F. M., J. Pizarro-Araya y G. E. Flores. 2009. Epigean Tenebrionids (Coleoptera: Tenebrionidae) from the Choros Archipelago (Coquimbo Region, Chile). Entomological News 120:125-130.

Barreda, V. y L. Palazzesi. 2007. Patagonian vegetation turnovers during the Paleogene-Early Neogene: Origin of arid-adapted floras. The Botanical Review 73:31-50.

Beeskow, A. M., H. del Valle y C. M. Rostagno. 1987. Los sistemas fisiográficos de la región árida y semiárida de la provincia del Chubut. SECYT (Secretaría de Ciencia y Técnica), Universidad de Buenos Aires. 173 p.

Bertiller, M., A. M. Beeskow y M. D. Irisarri. 1981. Caracteres florísticos y fisonómicos de la vegetación del Chubut. 2. Península Valdés e Istmo F. Ameghino. Contribución 41, Centro Nacional Patagónico. p. 1-20.

Botes, A., M. McGeoch y S. Chown. 2007. Ground-dwelling beetle assemblages in the northern Cape Floristic Region: 
Patterns, correlates and implications. Austral Ecology 32:210-224.

Bouchard, P., J. Lawrence, A. Davies y A. Newton. 2005. Synoptic classification of the world Tenebrionidae (Insecta: Coleoptera) with a review of family-group names. Annales Zoologici 55:499-530.

Bouza, P. J., M. Simón, J. Aguilar, H. del Valle y M. Rostagno. 2007. Fibrous-clay mineral formation and soil evolution in Aridisols of northeastern Patagonia, Argentina. Geoderma 139:38-50.

Brown, J. H. 1995. Macroecology. University of Chicago Press, Chicago. 269 p.

Cabrera, A. L. y A. Willink. 1980. Biogeografía de América Latina, segunda edición, Monografía 13. Serie de Biología. Secretaría General de la Organización de los Estados Americanos, Washington, D. C. 120 p.

Cepeda-Pizarro, J., J. Pizarro-Araya y H. Vásquez. 2005a. Composición y abundancia de artrópodos epígeos del Parque Nacional Llanos de Challe: impactos del ENOS de 1997 y efectos del hábitat pedológico. Revista Chilena de Historia Natural 78:635-650.

Cepeda-Pizarro, J., J. Pizarro-Araya y H. Vásquez. 2005 b. Variación en la abundancia de Artropoda en un transecto latitudinal del desierto costero transicional de Chile, con énfasis en los tenebriónidos epígeos. Revista Chilena de Historia Natural 78:651-663.

Chao, A. 1987. Estimating the population size for capturerecapture data with unequal catchability. Biometrics 43:783-791.

Cheli, G. H. 2009. Efectos del disturbio por pastoreo ovino sobre la comunidad de artrópodos epígeos en península Valdés (Chubut, Argentina). Tesis, Doctorado Universidad Nacional del Comahue - Centro Regional Universitario Bariloche, Río Negro. 256 p.

Cheli, G. H., J. C. Corley, O. Bruzzone, M. D. Brío, F. Martínez, N. M. Román y I. Ríos. 2010. The ground-dwelling arthropod community of Península Valdés in Patagonia, Argentina. Journal of Insect Science 10:50.

Cloudsley-Thompson, J. 2001. Thermal and water relations of desert beetles. Naturwissenschaften 88:447-460.

Codignotto, J. 2008. Península Valdés, entre el mar y la tierra. In Sitios de interés geológicos de la República Argentina, Anales 46, II, CSIGA (eds.). Instituto de Geología y Recursos Minerales, Servicio Geológico Minero Argentino, Buenos Aires. p. 683-696.

Colwell, R. K. 2005. EstimateS: Statistical estimation of species richness and shared species from samples,ver. 7.5, http:// purl.oclc.org/estimates; última consulta: 02.II.2010.

Colwell, R. K. y G. C. Hurtt. 1994. Nonbiological gradients in species richness and a spurious Rapoport effect. The American Naturalist 144:570-595.

Colwell, R. K. y D. Lees. 2000. The mid-domain effect: geometric constraints on the geography of species richness. Trends in Ecology and Evolution 15:70-76.

Cox, D. R. y P. A. Lewis. 1966. The statistical analysis of series of events. Methuen, London. 271 p.

Crawford, C. y M. Seely. 1987. Assemblages of surface-active arthropods in the Namib dunefield and associated habitats. Revue de Zoologie Africaine 101:397-421.

Deslippe, R. J., J. R. Salazar y Y. J. Guo. 2001. A darkling beetle population in West Texas during the 1997-1998 El Niño. Journal of Arid Environments 49:711-721.

Domínguez, M. C., S. Roig-Juñent, J. J. Tassin, F. C. Ocampo y G. E. Flores. 2006. Areas of endemism of the Patagonian steppe: an approach based on insect distributional patterns using endemicity analysis. Journal of Biogeography 33:1527-1537.

Donadio, E., S. Di Martinoa, M. Aubonea y A. Novarob. 2004. Feeding ecology of the yean hog-nosed skunk (Conepatus chinga) in areas under different land use in north-western Patagonia. Journal of Arid Environments 56:709-718.

Dozo, M. T., P. Bouza, A. Monti, L. Palazzesi, V. Barreda, G. Massaferro, R. A. Scasso y C. P. Tambussi. 2010. Late Miocene continental biota in Northeastern Patagonia (Península Valdés, Chubut, Argentina). Palaeogeography, Palaeoclimatology, Palaeoecology 297:100-109.

Dutilleul, P. 1993. Modifying the $t$ test for assessing the correlation between two spatial processes. Biometrics 49:305-314.

Elissalde, N., J. M. Escobar y V. Nakamatsu. 2002. Inventario y evaluación de pastizales naturales de la zona árida y semiárida de la Patagonia. EEA (Estación Experimental Agropecuaria), INTA (Instituto Nacional de Tecnología Agropecuaria), Chubut-PAN, 45 p.

Ferrer-Castán, D. y O. Vetaas. 2005. Pteridophyte richness, climate and topography in the Iberian Peninsula: comparing spatial and nonspatial models of richness patterns. Global Ecology and Biogeography 14:155-165.

Flores, G. E. 1998. Tenebrionidae. In Biodiversidad de artrópodos argentinos: una perspectiva biotaxonómica. J. J. Morrone y S. Coscarón (eds.). Sur, La Plata, Buenos Aires. p. 232-240.

Flores, G. E. 1999. Systematic revision and cladistic analysis of the Neotropical genera Mitragenius Solier, Auladera Solier and Patagonogenius gen. n. ( Coleoptera: Tenebrionidae). Entomologica Escandinavica 30:361-396.

Flores, G. E. 2004. Systematic revision and cladistic analysis of the Patagonian genus Platesthes (Coleoptera: Tenebrionidae). European Journal of Entomology 101:591608.

Flores, G. E. y J. Pizarro-Araya. 2006. The Andes mountain range as a vicariant event in the Pimeliinae (Coleoptera: Tenebrionidae) in southern South America. Cahiers Scientifiques 10:95-102. 
Flores, G. E., S. J. Lagos y S. A. Roig-Juñent. 2004. Artrópodos epígeos que viven bajo la copa del algarrobo (Prosopis Flexuosa) en la Reserva Telteca (Mendoza, Argentina). Multequina 13:71-90.

Flores, G. E., R. Carrara y G. H. Cheli. 2011. Three new Praociini (Coleoptera: Tenebrionidae) from Peninsula Valdés (Argentina), with zoogeographical and ecological remarks. Zootaxa 2965: 39-50.

Garcillán, P. P., E. Ezcurra y H. Riemman. 2003. Distribution of species richness of woody dryland legumes in Baja California, Mexico. Journal of Vegetation Science 14:473486.

Gardner, S. M., M. R. Cabido, G. R. Valladares y S. Diaz. 1995. The influence of habitat structure on arthropod diversity in Argentine semi-arid Chaco forest. Journal of Vegetation Science 6:349-356.

González-Megías, A., J. M. Gómez y F. Sánchez-Piñero. 2004. Effects of ungulates on epigeal arthropods in Sierra Nevada National Park (southeast Spain). Biodiversity and Conservation 13:733-752.

Gotelli, N. J., G. L. Entsminger, C. Rahbek, R. K. Colwell y G. R. Graves. 2007. BioGeoSim ver. 1.0. Acquired intelligence \& Kesey-Bear, Jericho, Vermont. , http://www. garyentsminger.com/biogeosim/biogeosim.htm; última consulta: 10.VI.2010.

Groom, M. J., G. K. Meffe y C. R. Carroll. 2006. Principles of conservation biology, tercera edition. Sinauer, Sunderland, Massachusetts. 699 p.

Groos, J. 2006. Nortest: tests for normality, ver.1.0. R package. University of Dortmund.

Haller, M., A. J. Monti y C. M. Meister. 2001. Hoja geológica 4363-I, península Valdés. Provincia del Chubut. Boletín 26. Instituto de Geología y Recursos Minerales, Servicio Geológico Minero Argentino, Buenos Aires. 25 p.

Hartley, A. 2003. Andean uplift and climate change. Journal of Geological Society 160:7-10.

Hijmans, R. J., L. Guarino, A. Jarvis, R. O'Brien y P. Mathur. 2005. DIVA-GIS ver. 5.4, http:www.diva-gis.org; última consulta: 05.III.2010.

Huston, M. A. 1994. Biological Diversity: The coexistence of species on changing landscapes. Cambridge University Press. 708 p.

Jenkins, D. G. y D. Rinne. 2008. Red herring or low illumination? The peninsula effect revisited. Journal of Biogeography 35:2128-2137.

Jiménez-Valverde, A. y J. Hortal. 2003. Las curvas de acumulación de especies y la necesidad de evaluar la calidad de los inventarios biológicos. Revista Ibérica de Aracnología 8:151-161.

Johnson, R. A. y P. S. Ward. 2002. Biogeography and endemism of ants (Hymenoptera: Formicidae) in Baja California, Mexico: a first overview. Journal of Biogeography 29:1009-
1026.

Koch, C. 1961. Some aspects of abundant life in the vegetationless sand of the Namib Desert dunes, positive psammotropism in Tenebrionid beetles. Journal of the South West Africa Scientific Society 15:8-34.

Kostadinoff, J. 1992. Estudio geofísico de la estructura de la península Valdés y los golfos nordpatagónicos. Revista de la Asociación Geológica Argentina 47:229-236.

Kulzer, H. 1955. Neue Tenebrioniden aus Südamerika. (Elfter Beitrag zur Kenntnis der Tenebrioniden). Entomologische Arbeiten aus dem Museum George Frey 6:479-485.

Kulzer, H. 1958. Monographie der südamerikanischen Tribus Praocini (Col.) (16 Beitrag zur Kenntnis der Tenebrioniden). Entomologische Arbeiten aus dem Museum Georg Frey 9:1-105.

Kulzer, H. 1963. Revision der südamerikanischen Gattung Nyctelia Latr. (Col. Teneb.) (24 Beitrag zur Kenntnis der Tenebrioniden). Entomologische Arbeiten aus dem Museum George Frey 14:1-71.

Kuschel, G. 1969. Biogeography and ecology of South American Coleoptera. In Biogeography and ecology in South America, vol. 2, E. J. Fittkau, J. Illies, H. Klinge, G. H. Schwabe y H. Sioli (eds.). Junk, The Hague. p. 709-722.

Lawton, J. H. y R. M. May. 1995. Extinction rates. Oxford University Press, New York. 233 p.

Lande, R. 1993. Risks of population extinction from demographic and environmental stochasticity and random catastrophes. The American Naturalist 142:911-927.

León, R., D. Bran, M. Collantes, J. Paruelo y A. Soriano. 1998. Grandes unidades de vegetación de la Patagonia extra andina. Ecología Austral 8:125-144.

MacArthur, R. H. y E. O. Wilson. 1967. The theory of island biogeography. Princeton University Press, New Jersey. 203 p.

Marcuzzi, G. 1951. Tenebrionidi come indicatori del clima. Rivista di Biologia di Perugia (n.s.) 43:399-437.

Molina, S. I., G. R. Valladares, S. Gardner y M. R. Cabido. 1999. The effects of logging and grazing on the insect community associated with a semi-arid Chaco forest in central Argentina. Journal of Arid Environments 42:29-42.

Morrone, J. J. 2006. Biogeographic areas and transition zones of Latin America and the Caribbean Islands based on panbiogeographic and cladistic analyses of the entomofauna. Annual Review of Entomology 51:467-494.

Morrone, J. J., S. A. Roig-Juñent y G. E. Flores. 2002. Delimitation of biogeographic districts in central Patagonia (southern South America), based on beetle distributional patterns (Coleoptera: Carabidae y Tenebrionidae). Revista del Museo Argentino de Ciencias Naturales n.s. 4:1-6.

Nabte, M., S. Saba y A. Monjeau. 2009. Mamíferos terrestres de la península Valdés: lista sistemática comentada. Mastozoología neotropical 16:109-120. 
Organismo Provincial de Turismo. 2001. Plan de Manejo del Área Protegida Sistema Península Valdés. Programa para la elaboración de los planes de manejo de las áreas naturales protegidas provinciales. Chubut. 203 p.

Pardiñas, U., P. Teta, S. Cirignoli y D. Podestá. 2003. Micromamíferos (Didelphimorphia y Rodentia) de norpatagonia extra andina, Argentina: taxonomía alfa y biogeografía. Mastozoología Neotropical 10:69-113.

Peterson, T. A., S. L. Egbert, V. Sánchez-Cordero y K. P. Price. 2000. Geographic analysis of conservation priority: Endemic birds and mammals in Veracruz, Mexico. Biological Conservation 93:85-94.

Peña, L. E. 1966. Catálogo de los Tenebrionidae (Coleoptera) de Chile. Entomologische Arbeiten aus dem Museum George Frey 17:397-453.

Polis, G. A. 1991. The ecology of desert communities. University of Arizona Press, Tucson. 456 p.

Preston, F. W. 1962. The canonical distribution of commonness y rarity: Part I. Ecology 43:185-215.

R Development Core Team. 2010. R: A language and environment for statistical computing, http://www.rproject.org.

Rahbek, C., N. J. Gotelli, R. K. Colwell, G. L. Entsminger, T. F. Rangel y G. R. Graves. 2007. Predicting continental-scale patterns of bird species richness with spatially explicit models. Proceedings of the Royal Society of Biological Sciences 274:165-174.

Rangel, T. F., J. A. Diniz-Filho y L. M. Bini. 2010. SAM: a comprehensive application for Spatial Analysis in Macroecology. Ecography 33:46-50.

Roig, F. A. 1998. La vegetación de la Patagonia. In Flora Patagónica. Colección Científica, M. M. Correa (ed.). Instituto Nacional de Tecnología Agropecuaria (INTA), Buenos Aires. p. 48-174.

Roig, F. A., S. A. Roig-Juñent y V. Corbalán. 2009. Biogeography of the Monte Desert. Journal of Arid Environments 73:164172.

Roig-Juñent, S. A. y G. E. Flores. 2001. Historia biogeográfica de las áreas áridas de América del Sur. In Introducción a la biogeografía en Latinoamérica: teorías, conceptos, métodos y aplicaciones, J. Llorente-Bousquets y J. J. Morrone (eds.). Facultad de Ciencias, Universidad Nacional Autónoma de México, México, D. F. p. 257-266.

Rostagno, C. M. 1981. Reconocimiento de suelos de península Valdés. Contribución 44. Centro Nacional Patagónico, Puerto Madryn, Chubut. p. 1-24.

Sackmann, P. y G. E. Flores. 2009. Temporal and spatial patterns of tenebrionid beetle diversity in NW Patagonia, Argentina. Journal of Arid Environments 12:1095-1102.

Simpson, G. G. 1964. Species density of North American recent mammals. Systematic Zoology 13:57-73.

Soberón, J. M. y J. B. Llorente. 1993. The use of species accumulation functions for the prediction of species richness. Conservation Biology 7:480-488.

Soriano, A. 1956. Los distritos florísticos de la provincia Patagónica. Revista de Investigaciones Agrícolas 10:323347.

Superina, M., F. Fernández-Campón, E. L. Stevani y R. Carrara. 2009. Summer diet of the pichi Zaedyus pichiy (Xenarthra: Dasypodidae) in Mendoza Province, Argentina. Journal of Arid Environments 73:683-686.

Sánchez-Piñero, F. y R. L. Aalbu. 2002. Tenebrionid Beetles. In A new island biogeography of the Sea of Cortes, T. J. Case, M. L. Cody y E. Ezcurra (eds.). Oxford University Press, New York. p. 129-153.

Súnico, A., H. del Valle, P. Bouza, L. Videla, C. Cano y A. Monti. 1994. Guía de Campo Península Valdés y CentroEste del Chubut. VII Reunión de campo del Comité Argentino de Investigación del Cuaternario (CADINQUA). Puerto Madryn, Chubut. 75 p.

Tschinkel, W. y J. Doyen. 1980. Comparative anatomy of the defensive glands, ovipositors y female genital tubes of tenebrionid beetles (Coleoptera). International Journal of Insect Morphology and Embryology 9:321-368.

Watt, J. C. 1974. A revised subfamily classification of Tenebrionidae (Coleoptera). New Zealand Journal of Zoology 1:381-452.

Zachariassen, K. E. 1996. The water conserving physiological compromise of desert insects. European Journal of Entomology 93:359-369. 